$7-28-2020$

\title{
Community-level interventions for pre-eclampsia (CLIP) in Pakistan: A cluster randomised controlled trial
}

\author{
Rahat Qureshi \\ Aga Khan University, rahat.qureshi@aku.edu \\ Sana Sheikh \\ Aga Khan University, sana.sheikh@aku.edu \\ Zahra Hoodbhoy \\ Aga Khan University, zahra.hoodbhoy@aku.edu \\ Sumedha Sharma \\ University of British Columbia, Vancouver, Canada \\ Marianne Vidler \\ University of British Columbia, Vancouver, Canada
}

See next page for additional authors

Follow this and additional works at: https://ecommons.aku.edu/pakistan_fhs_mc_women_childhealth_wc

Part of the Maternal and Child Health Commons, and the Public Health Education and Promotion

\section{Commons}

\section{Recommended Citation}

Qureshi, R., Sheikh, S., Hoodbhoy, Z., Sharma, S., Vidler, M., Payne, B. A., Ahmed, I., Ansermino, J. M., Bone, J., Bhutta, Z. A. (2020). Community-level interventions for pre-eclampsia (CLIP) in Pakistan: A cluster randomised controlled trial. Pregnancy Hypertension, 22, 109-118.

Available at: https://ecommons.aku.edu/pakistan_fhs_mc_women_childhealth_wc/142 


\section{Authors}

Rahat Qureshi, Sana Sheikh, Zahra Hoodbhoy, Sumedha Sharma, Marianne Vidler, Beth A. Payne, Imran Ahmed, J Mark Ansermino, Jeffrey Bone, and Zulfiqar Ahmed Bhutta 


\title{
Community-level interventions for pre-eclampsia (CLIP) in Pakistan: A cluster randomised controlled trial
}

\author{
Rahat N. Qureshi ${ }^{\mathrm{a}}$, Sana Sheikh ${ }^{\mathrm{a}}$, Zahra Hoodbhoy ${ }^{\mathrm{a}}$, Sumedha Sharma ${ }^{\mathrm{b}}$, Marianne Vidler ${ }^{\mathrm{b}, \mathrm{c}}$, \\ Beth A. Payne ${ }^{c}$, Imran Ahmed ${ }^{a}$, J. Mark Ansermino ${ }^{c}$, Jeffrey Bone ${ }^{b}$, Dustin T. Dunsmuir ${ }^{c}$, \\ Tang Lee ${ }^{\mathrm{b}}$, Jing Li ${ }^{\mathrm{b}}$, Hannah L. Nathan ${ }^{\mathrm{d}}$, Andrew H. Shennan ${ }^{\mathrm{d}}$, Joel Singer ${ }^{\mathrm{e}}$, Domena K. Tu ${ }^{\mathrm{b}}$, \\ Hubert Wong ${ }^{\mathrm{e}}$, Laura A. Magee ${ }^{\mathrm{b}, \mathrm{d}}$, Peter von Dadelszen ${ }^{\mathrm{b}, \mathrm{d}}$, Zulfiqar A. Bhutta ${ }^{\mathrm{a}, \mathrm{f}, *}$, the CLIP \\ Pakistan Working Group ${ }^{g}$ \\ ${ }^{a}$ Centre of Excellence, Division of Woman and Child Health, Aga Khan University, Stadium Road, P. O. Box 3500, Karachi 74800, Pakistan \\ ${ }^{\mathrm{b}}$ Department of Obstetrics and Gynaecology, Faculty of Medicine, University of British Columbia, Suite 930, 1125 Howe Street, Vancouver, BC V6Z 2K8, Canada \\ ${ }^{\mathrm{c}}$ Centre for International Child Health, University of British Columbia, 305-4088 Cambie Street, Vancouver, BC V5Z 2X8, Canada \\ ${ }^{\mathrm{d}}$ Department of Women and Children's Health, School of Life Course Sciences, Faculty of Medicine and Life Sciences, King's College London, 1 Lambeth Palace Road, \\ London SE1 7EH, UK \\ ${ }^{\mathrm{e}}$ Centre for Health Evaluation and Outcome Sciences, Providence Health Care Research Institute, University of British Columbia, 588 - 1081 Burrard Street, St. Paul's \\ Hospital, Vancouver, BC V6Z 1Y6, Canada \\ ${ }^{\mathrm{f}}$ Centre for Global Child Health, Hospital for Sick Children, 525 University Avenue, Suite 702, Toronto, ON M5G 2L3, Canada \\ ${ }^{g}$ the CLIP Pakistan Trial Working Group (Table S1)
}

\section{A R T I C L E I N F O}

\section{Keywords:}

Cluster randomized controlled trial

Pregnancy hypertension

Pakistan

Community engagement

Mobile health

Community health worker

\begin{abstract}
A B S T R A C T
Objectives: To reduce all-cause maternal and perinatal mortality and major morbidity through Lady Health Worker (LHW)-facilitated community engagement and early diagnosis, stabilization and referral of women with preeclampsia, an important contributor to adverse maternal and perinatal outcomes given delays in early detection and initial management.

Study design: In the Pakistan Community-Level Interventions for Pre-eclampsia (CLIP) cluster randomized controlled trial (NCT01911494), LHWs engaged the community, recruited pregnant women from 20 union councils (clusters), undertook mobile health-guided clinical assessment for preeclampsia, and referral to facilities after stabilization.

Main outcome measures: The primary outcome was a composite of maternal, fetal and newborn mortality and major morbidity.

Findings: We recruited 39,446 women in intervention $(\mathrm{N}=20,264)$ and control clusters $(\mathrm{N}=19,182)$ with minimal loss to follow-up ( $3 \cdot 7 \%$ vs. $4 \cdot 5 \%$, respectively). The primary outcome did not differ between intervention (26.6\%) and control (21.9\%) clusters (adjusted odds ratio, aOR, 1.20 [95\% confidence interval $0.84-$ $1.72] ; \mathrm{p}=0.31)$. There was reduction in stillbirths (0.89 [0.81-0.99]; $\mathrm{p}=0.03)$, but no impact on maternal death $(1.08[0.69,1.71] ; \mathrm{p}=0.74)$ or morbidity $(1.12$ [0.57, 2.16]; $\mathrm{p}=0.77)$; early $(0.95$ [0.82-1.09]; $\mathrm{p}=0.46)$ or late neonatal deaths (1.23 [0.97-1.55]; $\mathrm{p}=0.09)$; or neonatal morbidity $(1.22$ [0.77, 1.96]; $\mathrm{p}=0.40)$. Improvements in outcome rates were observed with 4-7 ( $\mathrm{p}=0.015)$ and $\geq 8(\mathrm{p}<0.001)$ (vs. 0) CLIP contacts.

Interpretation: The CLIP intervention was well accepted by the community and implemented by LHWs. Lack of effects on adverse outcomes could relate to quality care for mothers with pre-eclampsia in health facilities. Future strategies for community outreach must also be accompanied by health facility strengthening. Funding: The University of British Columbia (PRE-EMPT), a grantee of the Bill \& Melinda Gates Foundation (OPP1017337).
\end{abstract}

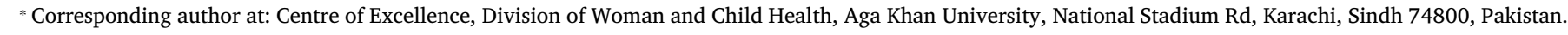
E-mail address: zulfiqar.bhutta@aku.edu (Z.A. Bhutta).
} 


\section{Introduction}

The Global Burden of Disease estimates that Pakistan, the world's sixth most populous country, endures the world's third highest burden of maternal mortality (348.6/100,000 live births [95\% uncertainty intervals (UI) 247-2-447.0]), stillbirth (27.6/1000 births [95\% UI 23.1$32 \cdot 8]$ ), and under-five child mortality (58.0/1000 live births [95\% UI 50-69]) [1,2]. In addition, Pakistan is recognised to have the highest neonatal mortality rate globally [3].

Such adversity is associated with sociocultural factors and health system-related barriers to accessing and receiving the best evidencebased care [4-7]. Among the barriers, delays in recognition of illness and seeking care contributed to $36 \%$ of maternal deaths in 2006 [7]. Formative research in Sindh Province reveals the importance of husbands and mothers-in-law as decision makers in health care utilization
[4]. Poor availability of transport, financial constraints, and limited mobility of women are important additional barriers to seeking care.

In response, the government of Pakistan has invested in health strategies and outreach services targeting poor and marginalised populations. The public health sector is comprised of primary-, secondary-, and tertiary-level health facilities, while the private health sector includes informal care providers (i.e., traditional birth attendants and spiritual healers) and formal medical clinics and/or hospitals. The mainstay of rural primary care is a cadre of community health workers (Lady Health Workers [8], LHWs). LHWs (currently almost 100,000) receive formal training for 15 months including didactic course work (3 months) and a 12-month practicum. They provide mainly maternal, newborn and child health (MNCH) preventative services, health promotion and referrals, as needed, to $100-150$ households, covering $70 \%$ of the rural populations in major provinces. LHWs receive monthly

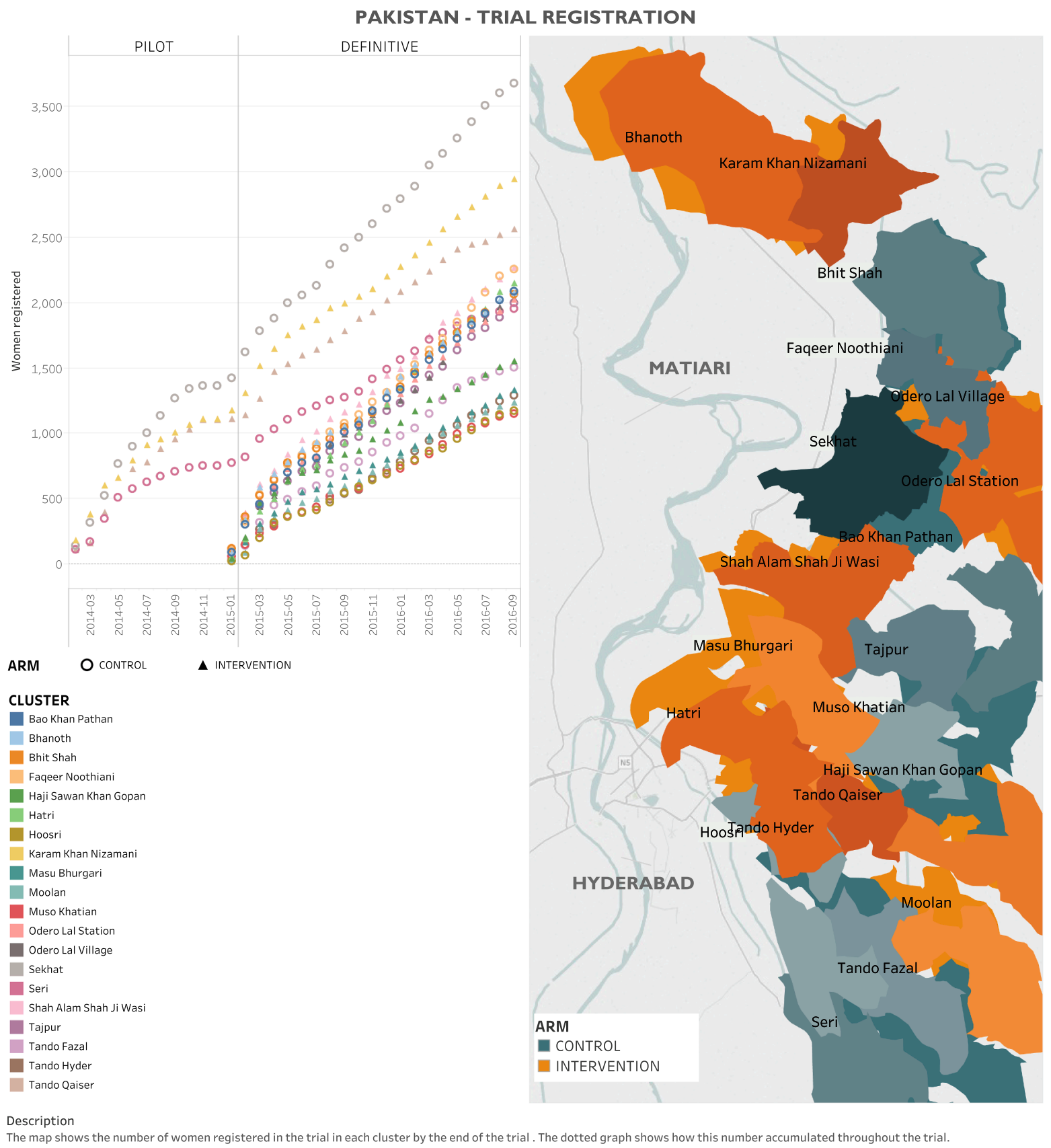

Fig. 1. Map of study area and enrollment by cluster. 
refreshers and replenishment of supplies in the primary care facilities of respective districts. On average, as noted in external evaluation [8], LHWs work on average five hours daily, visit an average 27 households per week and provide advice to an average 22 clients, usually women per week. Their current stipend is PKR 27,000 per month (US\$ 173).

Pregnancy hypertension is associated with maternal and perinatal morbidity and mortality risks worldwide, particularly in less-developed countries like Pakistan [9]. There is a general lack of understanding by women of the implications of having, and by health care providers of best evidence-based care of, pregnancy hypertension [4]. The detection and management of pregnancy hypertension is an important component of antenatal care (ANC) attendance [10]; its implementation reflects the quality of care in pregnancy. In resource-constrained environments such as Pakistan, standards against which health care delivery is benchmarked are often basic, such as whether BP or proteinuria were tested at least once in pregnancy, when each of these practices is to be delivered serially over the course of pregnancy [11]. Also, despite national policy, supplies of essential commodities (e.g., magnesium sulphate) are inadequate and few birthing facilities have established protocols for eclampsia management.

A number of innovations have been tested and introduced through the LHW programme, especially targeting perinatal and newborn care and childhood illnesses and immunisations [12-15]. However, they neither carry blood pressure (BP) measurement devices nor receive training about pregnancy hypertension management.

We hypothesised that training LHWs in community engagement (to raise awareness of, and education about, general pregnancy risks and, specifically, pregnancy hypertension) as well as community-based assessment, triage, and initial treatment of pre-eclampsia would improve maternal and perinatal outcomes. The aim of the Community-Level Interventions for Pre-eclampsia (CLIP) cluster randomised controlled trial (cRCT) in Sindh Province, Pakistan was to reduce maternal and perinatal mortality and major morbidity by $20 \%$ or more in intervention (vs. control) clusters, through a community-level intervention to address triage, (initial) treatment, and transport (to facility) of women with pregnancy hypertension.

\section{Methods}

The full protocol has been published (https://clinicaltrials.gov/ct2/ show/NCT01911494; Supplementary Appendix 1), and approved by the ethics review committees of the Aga Khan University (AKU, 2590Obs-ERC-13); University of British Columbia (UBC, H12-03497).

\subsection{Study setting and trial design}

The CLIP Pakistan trial was one of three independently powered cRCTs (others in India and Mozambique; all NCT01911494), and was conducted in Matiari and Hyderabad districts, Sindh Province, approximately $150 \mathrm{~km}$ north of Karachi (Fig. 1). Matiari is rural and located $25 \mathrm{~km}$ north of Hyderabad (population: $\approx 600,000$ ). Hyderabad is semi-urban (population: $\approx 1,883,000$; second most-populous district in Sindh). The consistent population density is 4.7 people/hectare. The vast majority of residents are Muslim, agriculturalists, and speak either Sindhi and/or Urdu. Literacy rates are 40\% (Matiari) to 50\% (Hyderabad). While women in the study region know about high blood pressure (BP) and seizures in pregnancy, they do not associate the two and they attribute hypertension to stress or weakness [4]. They have no Sindhi term for the most dangerous form of pregnancy hypertension, pre-eclampsia.

The pilot phase (from 7 February 2014 to 18 January 2015), aimed at assessing acceptability of the intervention, and transitioned to the definitive phase (from 19 January 2015 to 31 December 2016) based on $\geq 50 \%$ acceptance by women of health facility referral when indicated by the CLIP intervention (using data to 31 October 2014); no additional outcomes were assessed at the end of the pilot phase. Recruitment of new women ended on 30 September 2016, to allow time for recruited women to deliver by trial end on 31 December 2016.

\subsection{Participants}

Married pregnant women aged between 15 and 49 years were identified by the trial surveillance teams and written informed consent obtained for participation and quarterly data collection in surveillance visits.

\subsection{Intervention}

In the intervention clusters, the CLIP intervention package consisted of an annual group mobilisation meeting with about ten community leaders in each village, and LHW-led visits with eligible women in their homes, with a focus on the detection and management of pregnancy hypertension (Fig. S1). At the first home visit and again at around seven to eight months of pregnancy, LHWs engaged each participant herself, in her home, along with her immediate community (e.g., mother, mother-in-law, and female family or friends as she wished and were available). Activities were supported by culturally-appropriate pictograms developed with the LHW programme and describing maternal and perinatal risks associated with pregnancy hypertension, as well as awareness-raising and education about birth preparedness and complication readiness (e.g., prior permissions for care-seeking, transport plans, and funding care of obstetric emergencies).

In intervention areas, the LHWs were encouraged to provide domiciliary visits every four-weeks $(<28$ weeks), fortnightly (28-35 weeks), weekly ( $\geq 36$ weeks until birth), within 24 h of birth, and on postpartum days 3,7 , and 14 . Given the nature of tasks performed by LHWs, these visits were not mandated by LHW supervisors. Tasks were guided by the tablet-based PIERS On the Move (POM) mobile health (mHealth) application (app) [16], that included the miniPIERS (Pre-eclampsia Integrated Estimate of RiSk) time of disease risk stratification [17] with pictograms as visual prompts. Altogether 223 LHWs (and associated 13 Lady Health Supervisors) were trained to provide the CLIP intervention. The three-day didactic and participatory training consisted of BP measurement and use of the CLIP POM tool, with feedback and reinforcement intervention provided at regular monthly meetings.

In brief, the POM directed LHWs to first observe women to rule out emergency conditions (illustrated by pictograms) that would warrant immediate referral to a facility. In the absence of emergency conditions, LHWs were directed to measure BP twice, using standardised methods and a semi-automated digital device validated for use in pregnancy (Microlife BP 3AS1-2) [18]; a third measurement was required if either the systolic or diastolic BP differed by more than $10 \mathrm{mmHg}$ between the first two readings. From January 2015 to December 2016, LHWs also used a pulse oximeter to perform peripheral capillary oxygen saturation $\left(\mathrm{SpO}_{2}\right)$ at the first and any subsequently hypertensive visit [19].

The CLIP POM tool stratified women into one of three care pathways: usual ante-/postnatal care, non-urgent referral to a comprehensive emergency obstetric care (CEmOC) or higher facility within $24 \mathrm{~h}$, or urgent referral to a CEmOC or higher facility within four hours. In addition, women could be recommended to receive oral antihypertensive therapy (for severe hypertension) or intramuscular magnesium sulphate (for evidence of pre-eclampsia) (Fig. S1). In control clusters, women received routine ANC provided at PHCs by nurses and doctors and additional routine LHW domiciliary visits.

During 2015, health care providers from all secondary and tertiary, public and private facilities in the districts received three professional development sessions immediately prior to launching the trial and twice during the first year, focussed on evidence-based detection and management of pregnancy hypertension. 
Table 1

Baseline characteristics.

\begin{tabular}{|c|c|c|}
\hline & $\begin{array}{l}\text { Intervention } \\
(\mathrm{n}=20,235 \\
\text { pregnancies) }\end{array}$ & $\begin{array}{l}\text { Control } \\
\text { (n }=19,182 \\
\text { pregnancies) }\end{array}$ \\
\hline Clusters & 10 & 10 \\
\hline Population density (n/ha) * & $4 \cdot 1$ & $5 \cdot 3$ \\
\hline $\begin{array}{l}\text { Estimated annual birth rate/cluster } \\
\qquad(\mathrm{n} / 1000)\end{array}$ & 22 & 22 \\
\hline LHWs/cluster & $21[17,30]$ & $21[13,21.8]$ \\
\hline $\begin{array}{l}\text { Neonatal mortality ratio/1000 live } \\
\text { births (in previous } 12 \text { months } \\
\text { at baseline) * }\end{array}$ & $27[25 \cdot 1,36 \cdot 8]$ & $28 \cdot 3[20 \cdot 7,34 \cdot 1]$ \\
\hline Households & 16,373 & 15,569 \\
\hline Enrolled pregnancies & 20,235 & 19,182 \\
\hline $\begin{array}{l}\text { Nature of respondent was woman } \\
\text { herself }\end{array}$ & $19,737(97 \cdot 5 \%)$ & $18,612(97 \cdot 0 \%)$ \\
\hline Maternal age (year) * & $28[25,30]$ & $28[25,30]$ \\
\hline Married & $20,186(99 \cdot 8 \%)$ & $19,109(99 \cdot 6 \%)$ \\
\hline \multicolumn{3}{|l|}{ Religion } \\
\hline Muslim & $16,806(83 \cdot 1 \%)$ & $15,637(81 \cdot 5 \%)$ \\
\hline Hindu & $3374(16 \cdot 7 \%)$ & $3472(18 \cdot 1 \%)$ \\
\hline Other & $18(0 \cdot 1 \%)$ & $5(0.02 \%)$ \\
\hline $\begin{array}{l}\text { Women with } \geq 5 \text { years of schooling } \\
*\end{array}$ & $3971(19 \cdot 6 \%)$ & $3277(17 \cdot 1 \%)$ \\
\hline $\begin{array}{l}\text { Husbands with } \geq 5 \text { years of } \\
\text { schooling } \dagger\end{array}$ & $9532(47 \cdot 2 \%)$ & $8247(43 \cdot 2 \%)$ \\
\hline \multicolumn{3}{|l|}{ Obstetric history } \\
\hline $\begin{array}{l}\text { Parous * } \\
\text { Parity }\end{array}$ & $2[1,4]$ (missing & $\begin{array}{l}14,981(7 / 8 \cdot 4 \%) \\
2[1,4] \text { (missing }\end{array}$ \\
\hline \multicolumn{3}{|l|}{$\begin{array}{l}\text { Amongst previously pregnant } \\
\text { women }\end{array}$} \\
\hline Had previous stillbirth(s) $\%$ & $\begin{array}{l}2368(14 \cdot 6 \%) \\
\text { (missing } 11 \cdot 4 \%)\end{array}$ & $\begin{array}{l}2276(14 \cdot 6 \%) \\
\text { (missing } 11 \cdot 2 \%)\end{array}$ \\
\hline Had previous neonatal death(s) & $\begin{array}{l}2318(15 \cdot 2 \%) \\
\text { (missing } 0 \cdot 03 \%)\end{array}$ & $\begin{array}{l}2263(15 \cdot 4 \%) \\
\text { (missing } 0 \cdot 0 \%)\end{array}$ \\
\hline \multicolumn{3}{|l|}{$\begin{array}{l}\text { Delivery location in previous } \\
\text { pregnancy } \dagger\end{array}$} \\
\hline Home & $5051(31 \cdot 1 \%)$ & $5301(34 \cdot 1 \%)$ \\
\hline CEmOC (hospitals) & $7813(48 \cdot 0 \%)$ & $7522(48 \cdot 4 \%)$ \\
\hline Non-CEmOC facility & $3342(20 \cdot 6 \%)$ & $2645(17 \cdot 0 \%)$ \\
\hline ANC care sought $* *$ & $13,375(72.5 \%)$ & $14,153(81.6 \%)$ \\
\hline \multicolumn{3}{|l|}{ Current pregnancy } \\
\hline $\begin{array}{l}\text { Gestational age at enrolment } \\
\text { (week) }\end{array}$ & $19 \cdot 8[14 \cdot 1,26 \cdot 8]$ & $20 \cdot 6[14 \cdot 9,27 \cdot 4]$ \\
\hline Multiple pregnancy & $171(0 \cdot 8 \%)$ & $182(0 \cdot 9 \%)$ \\
\hline
\end{tabular}

Data presented as median [interquartile range] or number (\%). * Variables used as adjustment factors in analyses, chosen $a$ priori. $\dagger$ Variable used as adjustment factor in analyses, chosen following review of baseline data prior to knowledge of outcomes. $₹$ Not asked in pilot phase. ** ANC care sought includes only visits with a mandated blood pressure measurement, excluding LHW CLIP contacts and all other LHW contacts.

LHW $=$ Lady Health Worker. CEmOC $=$ Comprehensive emergency obstetric care.

\subsection{Baseline survey and surveillance}

In intervention and control clusters, 20 LHW programme-independent surveillance teams (140 individuals) were trained to undertake quarterly cross-sectional visits. Each team consisted of one male team leader (chaperone) and six female data collectors who visited all households and screened for married, pregnant women aged 15-49 years. Following informed consent, baseline individual- and household-level data were collected (Table 1). At each visit registered women were asked about antenatal care-seeking and birth preparedness, and postpartum, about adverse maternal ( $<42$ days postpartum), fetal, or neonatal events ( $<28$ days). When maternal or perinatal deaths were identified, an independent, experienced team was notified to perform verbal and social autopsies.

Data collection tools were developed iteratively with local investigators, derived from existing validated questionnaires where possible (e.g., WHO 2012 Verbal and Social Autopsy,[20] and translated to Sindhi (local language). Data were collected initially on paper forms (pilot phase) and then electronically using tablets (definitive phase). Data management protocols ensured data security by encryption, data tracking through user identification numbers and audit trails, and effective data synchronisation between within-cluster devices and the REDCap server (Vanderbilt University, USA) at UBC.

\subsection{Outcomes (Panel 1)}

The primary outcome was a composite of all-cause maternal, fetal, and newborn mortality and major morbidity. Pregnancies with multiple elements of the primary outcome were only counted once and no weighting was used. In addition to stillbirths, maternal and newborn deaths were estimated until 42 and 28 days after birth respectively. Maternal morbidity consisted of serious end-organ complications that included, but were not limited to, those related to pregnancy hypertension. Neonatal morbidity reflected problems related to early delivery or delivery of a baby in poor health. All deaths, as well as survived morbidities, were reviewed on a quarterly basis by an independent panel of physicians, masked to the cluster of origin, and excluding individuals who cared for the woman or baby under review.

The major secondary outcomes were birth preparedness and complication readiness, delivery in a facility able to provide emergency obstetric care, and proportion of facility births. Other outcomes included pre-eclampsia knowledge and awareness before birth, gestational age at birth, and mode of delivery.

\subsection{Sample size and randomisation}

Assuming an annual birth rate of $14 / 1000 /$ year in each cluster, a baseline incidence of maternal and perinatal mortality and morbidity of $9.6 \%$ in control clusters, $10 \%$ loss to follow-up, an intra-cluster co-efficient (ICC) of 0.002 , and a $20 \%$ reduction in all cause maternal, fetal, and newborn morbidity and mortality, an alpha of 0.05 , and $80 \%$ power, we calculated that we would require 20 clusters over two years. The data upon which the estimates were based on the published or available ranges and were provided by the site investigators based on recent surveillance data. A predetermined review of these sample size assumptions using data from the CLIP feasibility study, pilot and definitive trials was conducted independently by the statistical team (JB, TL, HW, JS) at study midpoint.

The unit of randomisation (cluster) was a Union Council (average population 32,000), and all associated villages and primary health centres (PHCs). The local team chose potential clusters based on comparability of health care infrastructure, surveillance team accessibility, and the absence of conflicting concurrent research activity. Restricted, stratified randomisation was undertaken according to population size. There were 3424 possible allocations of clusters to intervention or control, from which one was randomly selected. Two intervention and control clusters were chosen for the pilot phase based on their implementation readiness.

\subsection{Statistical analysis (full plan, Appendix 2)}

All pregnancies, except those of women who withdrew, were included in our primary, intention-to-treat analyses. The unit of analysis was pregnancy, classified as 'followed-up' (complete postpartum trial surveillance), 'lost-to-follow-up' (estimated date of delivery [EDD] at $\geq 3$ weeks before trial end but without follow-up data), and 'still-on follow-up' (EDD $<3$ weeks before trial end).

To mitigate potential bias due to differential loss-to and incomplete follow-up, the primary outcome of women who were lost-to, or still-on, follow-up was imputed ten times via multiple imputation by chain equations and Rubin's rules [21]. Imputation models were based on all primary analysis adjustment factors (see below) and interactions 
between trial arm and enrolment date (accounting for possible lag in intervention effects). In each imputed data set, the adjusted odds ratio (OR) for the intervention effect was estimated using a multi-level logistic regression including a random intercept term for each cluster. To improve precision, models were adjusted for variables at individual (i.e., age, parity, maternal primary education, previous delivery locations, and husband's primary education) and cluster-level (i.e., baseline neonatal mortality rate, LHW density, and population density). Sensitivity analysis including only complete cases was conducted with the same adjustment variables.

Further, analogous multi-level logistic regression models were fit to assess the sensitivity of the primary result to various other factors, including adjustment, missing data for a component of the primary outcome (when others were documented), gestational age at birth, and postpartum follow-up to < 42 days, as well as cluster-level aggregate analysis. Where sensitivity analyses included imputation, results were pooled (Rubin's rules) [21]. In an additional planned secondary analysis, we explored within the intervention arm, whether there was a relationship between our primary outcome and the intensity of CLIP contacts, categorised as $0,1-3,4-7$, or $\geq 8$, to reflect prior and current WHO recommendations for the frequency of antenatal contacts [22]; to account for factors related to the number of POM-guided visit and confounders, the analysis was adjusted for maternal age, basic education, parity, enrolment timing in the trial, and distance from the household to facility.

All analyses were repeated for each component of the primary outcome, albeit without imputation. Secondary and other outcomes were compared by baseline factor-adjusted multi-level models, as above. Statistical significance (two-sided) was set at $\mathrm{p}<0.05$ for the primary and $\mathrm{p}<0.01$ for other analyses, without adjustment for the interim analysis. R statistical software was used throughout.

An interim analysis was undertaken once complete data were received for women making up half of the planned sample size and reviewed by the data safety and monitoring board (DSMB). The stopping rule for both benefit and harm required an observed difference between groups associated with an alpha $<0.001$ (power 80\%). The DSMB reviewed all reported adverse events for participant safety.

\section{Results}

Between 7 February 2014 and 30 September 2016, 35,974 women $(39,417$ pregnancies) were recruited in 10 intervention (20,238 pregnancies) and 10 control (19,186 pregnancies) clusters. Of these pregnancies, 4357 (2231 in the intervention vs. 2126 in the control arm) were from the pilot phase. After accounting for 7 withdrawals, 20,235 and 19,182 pregnancies in intervention and control clusters, respectively, were included in the analysis (Fig. 2).

Pregnancies in intervention and control clusters were broadly similar at baseline, with most information coming directly from the participating woman herself (Table 1, Table S2). Women were generally in their late 20s; virtually all were married (some were either widowed or divorced between conception and recruitment) and self-identified as Muslim. Most had no basic education although almost half of their husbands did. Women were generally parous and when so, reported high rates of prior stillbirth $(\approx 15 \%)$ and neonatal death $(\approx 15 \%)$ (Table S2). Few women $(<1 \%)$ had multiple pregnancies. Generally, women were enrolled at $\approx 20$ weeks' gestation (Table 1 ).

LHW coverage varied widely between clusters (range: 9\% (Odero Lal Station) to $98 \%$ (Bhanoth)). There were 1368 community engagement sessions/cluster (total of 793 men's group sessions (7784 participants) and 16,691 LHW-led sessions in pregnant women's homes).

The visit number compliance target of 12 visits during pregnancy and the post-natal period was met in $5723(28.3 \%)$ pregnancies. Of the total 58,174 visits, 10,529 women $(11,399$ pregnancies [56.6\%]) received $\geq 1$ LHW-provided POM visit, for a median of 5 [IQR 3, 7] visits per pregnancy when received. Most visits were antenatal (40,264
[69.2\%]). Compliance with BP measurement at all visits, and proteinuria testing during first and all hypertensive visits, was almost uniform (99.9\% and $98.4 \%$, respectively).

POM-guided advice for referrals, whether non-urgent $(2.5 \%$ of pregnancies) or urgent (1.4\% of pregnancies), were accepted $77 \%$ of the time. Non-urgent referral (2.5\%) was for non-severe hypertension. Urgent referral (1.4\%) was most commonly for severe hypertension $(153 / 218,70 \cdot 2 \%)$ or absent fetal movements in the preceding $24 \mathrm{~h} \mathrm{(24/}$ $218,11.0 \%$ ), and accepted $84.9 \%$ of the time. Intramuscular magnesium sulphate (for pre-eclampsia) was recommended in $166(0.3 \%)$ of POM visits and accepted in 96 visits (58.3\% of the time). Oral methyldopa (for severe hypertension) was recommended in $153(0.3 \%)$ visits and accepted in 148 visits (96.8\% of the time). Details of the indications for referral according to the CLIP triggers, and LHW actions, can be found in Table S3 and Figs. S1 and S2.

There was no significant difference in $\geq 4$ ANC visits for women in intervention (44.6\%) vs. control (31.7\%) clusters (aOR $1.8495 \% \mathrm{CI}$ [0.89-3.83]; $\mathrm{p}=0.032$ ).

The composite primary outcome (one or more element) occurred in more than $20 \%$ of pregnancies, related primarily to major maternal morbidity and fetal/newborn death $(\approx 10 \%$ each) (Table 2$)$. The primary outcome did not differ between intervention and control clusters overall (aOR 1.20 [0.84-1.72]; p = 0.31) (Table 2), or by cluster (Table S4). Major maternal morbidity was 37 times more common than was maternal death $(2 \cdot 7 / 1000$ identified pregnancies). Stillbirths ( $\geq 20$ weeks) and neonatal death each complicated $\approx 5 \%$ of pregnancies. Overall, there were no significant differences between the groups for the most common major maternal morbidities (sepsis (6.8\%), followed by antepartum haemorrhage (2.9\%) and blood transfusion $(1 \cdot 0 \%))$. The most common major neonatal morbidity was feeding difficulty (3.1\%), followed by lethargy (3.0\%) and hypothermia (1.8\%).

There was no difference in intervention (vs. control) clusters for birth preparedness $(42 \cdot 4 \%$ vs. $29 \cdot 1 \%$, respectively; aOR 2.41 [0.67$8 \cdot 67] ; \mathrm{p}=0.08)$ or pre-eclampsia knowledge and awareness (16.8\% vs. $14 \cdot 7 \%$, respectively; aOR 1.11 [0.33-3.69]; $\mathrm{p}=0.83$ ).

Within intervention clusters, women who received both 4-7 and at least eight POM guided contacts experienced fewer adverse outcomes compared with women who received no contacts $(28 \cdot 8 \%$ (4-7) and $23.2 \%(\geq 8)$ vs $30 \cdot 5 \%$ (0), respectively (aOR 0.89 [95\% CI 0.81-0.98]; $\mathrm{p}=0.015$ and 0.66 [95\% CI 0.58-0.76]; p < 0.001, respectively) (Table 3; Fig. S3). Women with 1-3 POM-guided contacts did not have apparent benefit compared with those without POM contacts (Table 3; Fig. S3). Rates of stillbirth were higher in the 1-3 contact group (7.4\% vs 5.7\%; aOR 1.50 [95\% CI 1.24-1.82]; p < 0.001), with a trend to more neonatal deaths $(\mathrm{p}=0.029)$, compared with those receiving no contacts (Table 3; Fig. S3).

A single adverse event was reported due to administration of the intervention to a non-pregnant woman who had not been enrolled (Table 4). The event was identified promptly, and trial activities paused during steering committee review of events. The DSMB concluded that no harm resulted from these actions.

\section{Discussion}

In the primary, pre-defined analysis, the CLIP Pakistan Trial intervention did not have a significant impact on either the composite outcome of maternal, fetal, newborn mortality and severe morbidity, or individual components thereof. This was an effectiveness trial with functional public sector LHWs, who despite busy workloads were able to undertake the recommended number of home visits in nearly half the cases. The intervention was reasonably well received by families though the overall reported morbidities detected by POM were low.

The finding of a positive effect of contact intensity of at least four contacts, and more so with at least eight contacts, provides data to support both the previous and current WHO recommendations. [22] While the current WHO recommendations are based on improved 


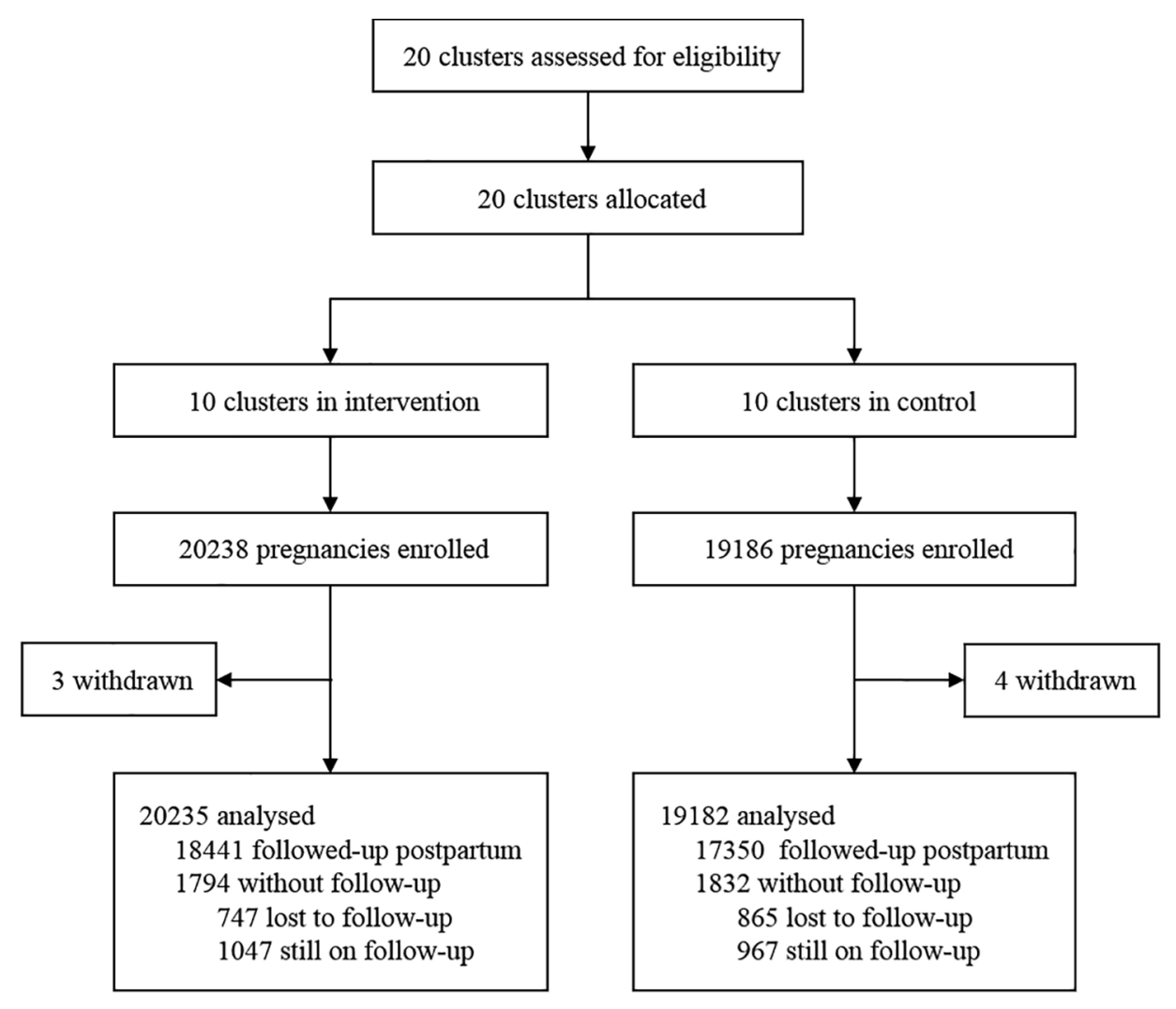

Lost to follow-up = Pregnancies that were 6 weeks postpartum more than one surveillance cycle ( $>4$ months) from the end of the trial.

Still on follow-up = Pregnancies that were antepartum at their last surveillance within one surveillance cycle $(<4$ months) from the end of the trial, or, pregnancies that were 6 weeks postpartum within one surveillance cycle ( $<4$ months) from the end of the trial.

Fig. 2. Trial profile - Intervention vs. control allocation clusters.

perinatal outcomes, our findings (vs. 0 contacts) imply both maternal and perinatal outcomes improved (Table 3 and Fig. S2). Women with 0 contacts were likely to live closer to facilities than those with 1-3 contacts; these latter women had higher rates of fetal and neonatal mortality. Compared with women who received 1-3 contacts, other than maternal death and neonatal morbidity, all components of the primary outcome were significantly reduced with at least four contacts.

Our study design and operational plan had several strengths. We successfully engaged civil society, from women themselves, their families, faith leaders, and government ministries. Complementing the existing health care system by task-sharing, the LHWs used the POM mHealth platform to conduct independent antenatal and postnatal visits and collect their findings. Our community-based data collection procedures resulted in a low $(<5 \%)$ loss to follow-up despite enrolment of nearly 40,000 pregnancies. However, including only married women with settled addresses excluded single and internally displaced women at higher risk of adverse outcomes; the trial was conducted following the significant internal displacement related to the 2010 Indus River floods.

Notwithstanding the careful design of the intervention, the effect could have been limited by a number of factors. There were insufficient LHWs to deliver the intervention to reach all sections of the population, as demonstrated by $<80 \%$ of women receiving a POM visit and $<80 \%$ compliance with the pre-specified schedule of visits when they occurred. The LHWs have significant tasks related to participation in the periodic polio eradication campaigns which overall took almost a third of their time. The annual community engagement may not have been sufficient to induce awareness and behaviour change. We had based our threshold for treatment and referral due to systolic hypertension to increase generalisability where functioning sphygmomanometers and training are lacking; in retrospect, this may have limited the impact of the CLIP intervention as CLIP data have shown that non-severe hypertension was predominately isolated diastolic hypertension [23]; the advent of low-cost, validated (in pregnancy and pre-eclampsia), semiautomated BP devices has mitigated some of the BP measurement concerns [18]. However, despite acceptance and ready application in training programmes, the lack of impact of three technological innovations (BP measurement device, POM and pulse oximetry) also raises the question of the role of technology in increasing community engagement and effectiveness of these frontline workers. The fact that there was no impact on knowledge and awareness of pre-eclampsia and danger signs among participating women suggests that these domiciliary interactions by LHWs may not have been effective. Finally, despite recruiting almost 36,000 women (and almost 40,000 pregnancies), we may have been underpowered to find a difference between trial arms as power is determined by cluster number and the betweencluster outcome rate variability was greater than anticipated (i.e., ICC 0.044).

We undertook community-ascertainment of outcomes. While measures of self-reported maternal, fetal, and neonatal outcomes have been validated in other studies [24], misreporting may have occurred. Also, the process of community engagement may have sensitised intervention cluster women and their families to disproportionately report adverse events which could explain the higher reported event rates in the intervention clusters in the first surveillance quarter. We were unable to use physical examination or chart review to confirm diagnoses, by virtue of the personnel who provided care (i.e., LHWs) and the location of that care (i.e., in the community where there is no health record). However, to mitigate this, a group allocation-masked physician review to adjudicate every reported maternal, fetal or newborn death or major 
Table 2

Primary outcome.

\begin{tabular}{|c|c|c|c|c|}
\hline & Intervention ( $\mathrm{n}=20,235$ pregnancies) & Control ( $\mathrm{n}=19,182$ pregnancies $)$ & Adjusted OR* & $\mathrm{p}$-value \\
\hline Pregnancies with postpartum surveillance & 18,419 & 17,335 & & \\
\hline Infants & 18,543 & 17,488 & & \\
\hline Composite primary outcome $\dagger$ & $5373(26 \cdot 6 \%)$ & $4187(21 \cdot 8 \%)$ & $1 \cdot 20[0 \cdot 84-1 \cdot 72]$ & $0 \cdot 31$ \\
\hline Maternal mortality & $55(0 \cdot 3 \%)$ & $51(0 \cdot 3 \%)$ & $1.08[0.69-1 \cdot 71]$ & $0 \cdot 74$ \\
\hline $\begin{array}{l}\text { Maternal morbidity } \\
\text { (including maternal deaths) }\end{array}$ & $2196(10 \cdot 9 \%)$ & $1707(8.9 \%)$ & $1 \cdot 12[0 \cdot 57-2 \cdot 16]$ & $0 \cdot 77$ \\
\hline Obstetric sepsis & $1477(7.3 \%)$ & $1191(6 \cdot 2 \%)$ & & \\
\hline Antepartum haemorrhage & $643(3.2 \%)$ & $487(2 \cdot 5 \%)$ & & \\
\hline Blood transfusion & $240(1.2 \%)$ & $168(0.9 \%)$ & & \\
\hline Stroke & $58(0.3 \%)$ & $79(0 \cdot 4 \%)$ & & \\
\hline Fistula & $56(0.3 \%)$ & $39(0 \cdot 2 \%)$ & & \\
\hline Seizure & $32(0.2 \%)$ & $18(0 \cdot 1 \%)$ & & \\
\hline Maternal coma & $27(0.1 \%)$ & $25(0 \cdot 1 \%)$ & & \\
\hline Cardiopulmonary resuscitation & $11(0.1 \%)$ & $17(0 \cdot 1 \%)$ & & \\
\hline Mechanical ventilation & $11(0 \cdot 1 \%)$ & $9(0 \cdot 0 \%)$ & & \\
\hline Disseminated intravascular coagulation & $4(0 \cdot 0 \%)$ & $8(0.0 \%)$ & & \\
\hline Dialysis & $2(0.0 \%)$ & $3(0.0 \%)$ & & \\
\hline Interventions for major postpartum haemorrhage & $2(0.0 \%)$ & $5(0.0 \%)$ & & \\
\hline Perinatal mortality and late neonatal death & $1942(9 \cdot 6 \%)$ & $1906(9 \cdot 9 \%)$ & $0.95[0 \cdot 86-1 \cdot 03]$ & $0 \cdot 22$ \\
\hline Stillbirth & $935(4 \cdot 6 \%)$ & $951(5 \cdot 0 \%)$ & $0.89[0.81-0.99]$ & $0 \cdot 03$ \\
\hline Early neonatal death & $819(4 \cdot 0 \%)$ & $818(4 \cdot 3 \%)$ & $0.95[0 \cdot 82-1 \cdot 09]$ & $0 \cdot 46$ \\
\hline Late neonatal death & $197(1 \cdot 0 \%)$ & $150(0 \cdot 8 \%)$ & $1 \cdot 23[0 \cdot 97-1 \cdot 55]$ & $0 \cdot 09$ \\
\hline Neonatal morbidity & $2012(11 \%)$ & $1250(7 \cdot 2 \%)$ & $1 \cdot 22[0 \cdot 77-1 \cdot 96]$ & $0 \cdot 40$ \\
\hline \multicolumn{5}{|l|}{ Neonatal morbidity (excluding deaths) } \\
\hline Feeding difficulty & $844(4 \cdot 2 \%)$ & $385(2.0 \%)$ & & \\
\hline Lethargy & $585(2.9 \%)$ & $609(3.2 \%)$ & & \\
\hline Hypothermia & $405(2.0 \%)$ & $288(1.5 \%)$ & & \\
\hline Jaundice & $385(1.9 \%)$ & $389(2.0 \%)$ & & \\
\hline Breathing difficulty & $273(1 \cdot 3 \%)$ & $223(1.1 \%)$ & & \\
\hline Seizure & $253(1.3 \%)$ & $261(1.4 \%)$ & & \\
\hline Umbilical cord infection & $284(1 \cdot 4 \%)$ & $241(1.3 \%)$ & & \\
\hline Coma & $149(0.7 \%)$ & $109(0.6 \%)$ & & \\
\hline Bleeding & $82(0 \cdot 4 \%)$ & $44(0.2 \%)$ & & \\
\hline Central nervous system-related morbidity & $12(0 \cdot 1 \%)$ & $14(0.1 \%)$ & & \\
\hline Skin infection & $2(0 \cdot 0 \%)$ & $1(0 \cdot 0 \%)$ & & \\
\hline
\end{tabular}

Data presented as number (\%) or number only. *Adjusted odds ratio presented as odds ratio [95\% confidence interval]; adjusted for individual-level factors (maternal age, parity, maternal education, marital status, husband's education, delivery location), and cluster-level factors (population density, baseline study neonatal mortality rate, healthcare worker density). $\uparrow$ Defined as one/more of maternal morbidity or mortality, stillbirth, neonatal mortality, or neonatal morbidity.

$\mathrm{OR}=$ odds ratio.

morbidity, was performed. Recall bias risks were minimised by conducting quarterly surveillance cycles.

Our population-based estimates of maternal, fetal, and neonatal mortality tended to be higher than those previously published for Pakistan. Most previous estimates for Pakistan have been facility-based. Our maternal mortality estimate was 323/100,000 livebirths (compared with 259 (AMANHI cohort of 31,114 pregnancies in Matiari) [25] and 299 (secondary analysis of the WHO Multicountry Survey 2011) [26]. For stillbirths, our estimate was 57.5/1000 livebirths (compared with 42.8 and $27 \cdot 6 / 1000$ livebirths for Matiari and nationally, respectively). [2,25] Our estimate of neonatal mortality was 60.4/1000 livebirths (compared with 46.9 and 55/1000 livebirths for Matiari and nationally [Pakistan DHS 2011-12], respectively [27]. For stillbirths, these differences may have reflected more comprehensive and accurate ascertainment of outcomes that may have been previously misclassified as miscarriages in the community, and that may not have come to the attention of those providing facility-based care.

The comparability of our community-ascertained rates of morbidity are more difficult to assess, as comparable published data are limited and facility-based. Our incidence of maternal sepsis (7.4\%) was much higher than the recorded incidence of sepsis recorded in tertiary facilities in Punjab and Sindh (1.3\% in all women) [28]; however, this definition of sepsis (comprising abortion-related infection, puerperal endometritis, pyelonephritis, influenza-like illness, other systemic infections) excluded organ dysfunction whereas our broad definition of sepsis (fever and one of: abdominal/uterine tenderness, foul smelling vaginal discharge/lochia, productive cough and shortness of breath, dysuria or flank pain, headache and neck stiffness) could have captured associated complications related to organ dysfunction. The incidence of antepartum haemorrhage in our trial $(3.2 \%)$ was within the range reported in facility-based studies in Peshawar (3.0\%) [29] or Hyderabad (5.4\%). We found no comparable regional data on population level estimates of neonatal morbidities.

A crucial factor limiting the effect on mortality and severe morbidity could also be the design which did not include facility-based interventions and care. Our hypothesis was that implementing communitylevel evidence-based care would reduce hypertension-related (and potentially, all cause) maternal and perinatal mortality and major morbidity, by addressing the 'three delays' in triage, transport, and treatment. While we did undertake staff capacity enhancement throughout the district, we did not specifically put measures in place to improve quality of care. Poor-quality facility care and availability of key commodities could have limited the beneficial effect of community-level intervention on outcomes and negatively influenced community demand; perceived poor quality of care at public hospitals is a significant barrier to seeking care [4], and health care providers in Sindh have gaps in their knowledge about the aetiology, diagnosis, and treatment of pre-eclampsia [6].

Finally, while the individual components of the complex CLIP intervention were evidence-based, how these components interacted with one another and moderated any impact of the intervention remains unclear but will be the subject of pre-determined secondary analyses.

The CLIP trial further confirms the ability of LHWs to task-share important aspects of maternity care, with favourable effects on 
outcomes, as observed for newborns and children with other community-level interventions in Pakistan [13,30]. For example, neonatal mortality was reduced by LHW-guided care (basic newborn resuscitation and identification and treatment of suspected neonatal respiratory infections (RR 0.80 [0.68, 0.93]; $p=0.005$ ) [13] or LHW-led rural group sessions focussed on promotion of antenatal care and maternal health education, use of clean delivery kits, facility births, immediate newborn care, identification of danger signs, and promotion of care seeking (RR 0.85 [0.76, 0.96]; $p=0.02$ ) [30]. Other community-level interventions in Pakistan have noted similar non-specific temporal improvements to those that we observed, and also highlighted the limitation of technology- and home-based care strategies for reducing newborn mortality when separated from facility enhancement [13].

\section{Conclusions}

Pakistan is striving to meet global targets for the sustainable development goals to improve maternal, fetal, and newborn outcomes while tackling the challenge of limited human resources, especially skilled medical professionals in its rural and remote populations. This was the genesis of our study to evaluate what could be done to mitigate the morbidity and mortality associated with pre-eclampsia. The CLIP Pakistan Trial demonstrates that an existing cadre of public sector community health workers can use technology to provide accurate and objective referral advice in pregnancy and engage and mobilize communities. However, as implemented, the intervention did not improve outcomes. To achieve benefits on mortality and severe morbidity, these efforts must be complemented with facility-based strengthening of quality clinical care and facilitation of community-to-facility referral to achieve maximal impact on the unacceptably-high burden of death and morbidity in such settings.

\section{Contributors}

RQ, ZAB, PvD, LAM, BAP, JMA, AS, HN conceptualised the Trial and the components of the intervention. ZAB, RQ, SS, ZH, BAP, MV, SS coordinated activities and monitoring of the Trial. IA, JL, TL, DT, DD were responsible for data collection, transfer and data cleaning. JS, HW, TL, JB performed the analyses. PvD, LAM, SS wrote the first draft of the manuscript. All authors provided feedback and review of the manuscript.

\section{Declaration of Competing Interest}

The authors declare that they have no known competing financial interests or personal relationships that could have appeared to influence the work reported in this paper.

\section{Acknowledgements}

We would like to thank the women and their families who contributed data to this trial, in addition to the LHWs who delivered care to the most vulnerable women in their communities.

\section{Role of the funding source}

The Bill \& Melinda Gates Foundation supported the University of British Columbia (PRE-EMPT) as a grantee (OPP1017337), with a subcontract to the Aga Khan University for this work (F13-02778). Following input into trial design, the Bill \& Melinda Gates Foundation had no role in data collection, analysis, or interpretation, or writing of the report. The corresponding author (ZAB) and PRE-EMPT principal investigator (PvD) had full access to all data in the study and are the guarantors with final responsibility for the decision to submit for publication. 
Table 4

Secondary, safety, and other outcomes.

Intervention ( $\mathrm{n}=20,235$

pregnancies)
Control ( $\mathrm{n}=19,182$

pregnancies)

\section{Secondary outcomes}

Birth preparedness and complication readiness $\dagger$

Proportion of facility births

Birth at a CEmOC facility

Other outcomes

Safety outcomes

SAEs unrelated to intervention

Adverse events

Transport-related injury or death

Injection site haematoma/infection after community administration of IM MgSO4

Injection site complications after any administration of IM MgSO4

Respiratory depression, coma or death during transport following incommunity MgSO4

Maternal sBP $<110 \mathrm{mmHg}$ on facility arrival following in-community methyldopa

Deliveries (all birth outcomes)

Miscarriage ${ }^{* * *}$

Livebirth

Deliveries (excluding miscarriage)

Gestational age at delivery(week)

Deliveries $<37$ weeks

Deliveries $<34$ weeks

Missing

$\begin{array}{ll}8532(42 \cdot 2 \%) & 5580(29 \cdot 1 \%) \\ 13,517(66 \cdot 8 \%) & 12,709(66 \cdot 3 \%) \\ 3949(65 \cdot 5 \%) & 3645(68 \cdot 3 \%)\end{array}$

$3645(68 \cdot 3 \%)$

$0.96[0 \cdot 71-1 \cdot 29] \quad 0.695$

$0.90 .60-1.34] \quad 0.482$

$\begin{array}{llll}0(0 \cdot 0 \%) & 0(0 \cdot 0 \%) & \text { NA } & \text { NA } \\ 0 / 305(0 \cdot 0 \%) & \text { NA } & \text { NA } & \text { NA } \\ 0 / 73(0 \cdot 0 \%) & \text { NA } & \text { NA } & \text { NA } \\ \text { NA } & \text { NA } & \text { NA } & \text { NA } \\ \text { NA } & \text { NA } & \text { NA } & \text { NA } \\ & & & \text { NA } \\ \text { NA } & \text { NA } & \text { NA } & \\ & & & - \\ 18,418 & 17,331 & - & 0 \cdot 476 \\ 559(3 \cdot 0 \%) & 500(2 \cdot 9 \%) & 1 \cdot 10[0 \cdot 78-1 \cdot 57] & \\ 16,943(83 \cdot 7 \%) & 15,913(83 \cdot 0 \%) & & 0 \cdot 708 \\ 17,862 & 16,835 & & 0 \cdot 883 \\ 38 \cdot 7[36 \cdot 0-41 \cdot 0] & 38 \cdot 6[35 \cdot 7-41 \cdot 0] & 0.96[0 \cdot 72-1 \cdot 29] & 0 \cdot 752 \\ 5475(29 \cdot 7 \%) & 5543(32 \cdot 0 \%) & 0.93[0 \cdot 73-1 \cdot 19] & 0 \cdot 450 \\ 2432(13 \cdot 2 \%) & 2466(14 \cdot 2 \%) & & \\ 1112(6 \cdot 0 \%) & 937(5 \cdot 4 \%) & & \end{array}$

Data presented as median [interquartile range] or number (\%) or number only. *Odds ratio adjusted for individual-level (i.e., maternal age, parity, maternal primary

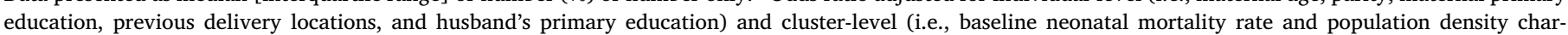

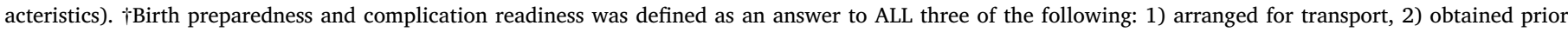
permission to seek emergency care, and 3) saved money for obstetric care.

$\mathrm{OR}=$ odds ratio. $\mathrm{CEmOC}=$ comprehensive emergency obstetric care $\mathrm{sBP}=$ systolic blood pressure.

\section{Appendix A. Supplementary data}

Supplementary data to this article can be found online at https:// doi.org/10.1016/j.preghy.2020.07.011.

\section{References}

[1] Institute for Health Metrics and Evaluation. Health related SDGs - Viz Hub. Available at: https://vizhub.healthdata.org/sdg/, 2017 (accessed 8 October 2019).

[2] H. Wang, Z. Bhutta, M. Coates, M. Coggeshall, L. Dandona, K. Diallo, et al., Global, regional, national, and selected subnational levels of stillbirths, neonatal, infant, and under-5 mortality, 1980-2015: a systematic analysis for the Global Burden of Disease Study 2015, Lancet 388 (10053) (2015) 1725-1774.

[3] UNICEF Data, Neonatal mortality. Available at: https://data.unicef.org/topic/childsurvival/neonatal-mortality/, 2019 (accessed 8 October 2019).

[4] R.N. Qureshi, S. Sheikh, A.R. Khowaja, Z. Hoodbhoy, S. Zaidi, D. Sawchuck, M. Vidler, Z.A. Bhutta, P. von Dadeslzen, Health care seeking behaviours in pregnancy in rural Sindh, Pakistan: a qualitative study, Reprod. Health 13 (S1) (2016), https://doi.org/10.1186/s12978-016-0140-1.

[5] A.R. Khowaja, R.N. Qureshi, D. Sawchuck, O.T. Oladapo, O.O. Adetoro, E.A. Orenuga, M. Bellad, A. Mallapur, U. Charantimath, E. Sevene, K. Munguambe, H.E. Boene, M. Vidler, Z.A. Bhutta, P. von Dadelszen, The feasibility of community level interventions for pre-eclampsia in South Asia and Sub-Saharan Africa: a mixed-methods design, Reprod. Health 13 (S1) (2016), https://doi.org/10.1186/ s12978-016-0133-0.

[6] S. Sheikh, R.N. Qureshi, A.R. Khowaja, R. Salam, M. Vidler, D. Sawchuck, P. von Dadelszen, S. Zaidi, Z. Bhutta, Health care provider knowledge and routine management of pre-eclampsia in Pakistan, Reprod. Health 13 (S2) (2016), https://doi. org/10.1186/s12978-016-0215-Z.

[7] Z.A. Bhutta, A. Hafeez, A. Rizvi, N. Ali, A. Khan, F. Ahmad, et al., Reproductive, maternal, newborn, and child health in Pakistan: challenges and opportunities, Lancet 381 (9884) (2013) 2207-2218.

[8] A. Hafeez, B.K. Mohamud, M.R. Shiekh, S.A.I. Shah, R. Jooma, Lady health workers programme in Pakistan: challenges, achievements and the way forward, J. Pak. Med. Assoc. 61 (3) (2011) 210-215.

[9] S. Perveen, Frequency and impact of hypertensive disorders of pregnancy, J. Ayub Med. Coll. Abbottabad 26 (4) (2014) 518-521.

[10] A. Oakley, Origins and development of antenatal care, in: M. Enkin, I. Chalmers (Eds.), Effectiveness and Satisfaction with Antenatal Care, Cambridge University Press, Cambridge, 1982, pp. 1-21.

[11] UNICEF Data. Antenatal care coverage. Available at: https://data.unicef.org/topic/ maternal-health/antenatal-care/, June 2018 (accessed September 2018).

[12] J.M. Smith, S. Currie, T. Cannon, D. Armbruster, J. Perri, Are national policies and programs for prevention and management of postpartum hemorrhage and preeclampsia adequate? A key informant survey in 37 countries, Glob. Health Sci. Pract. 2 (3) (2014) 275-284.

[13] S. Soofi, S. Cousens, A. Turab, Y. Wasan, S. Mohammed, S. Ariff, et al., Effect of provision of home-based curative health services by public sector health-care providers on neonatal survival: a community-based cluster-randomised trial in rural Pakistan, Lancet Glob Health 5 (8) (2017) e796-e806.

[14] M.A. Habib, S. Soofi, S. Cousens, S. Anwar, N.U. Haque, I. Ahmed, et al., Community engagement and integrated health and polio immunisation campaigns in conflict-affected areas of Pakistan: a cluster randomised controlled trial, Lancet Glob. Health 5 (6) (2017) e593-e603.

[15] S. Soofi, S. Ahmed, M.P. Fox, W.B. MacLeod, D.M. Thea, S.A. Qazi, et al., Effectiveness of community case management of severe pneumonia with oral amoxicillin in children aged 2-59 months in Matiari district, rural Pakistan: a cluster-randomised controlled trial, Lancet 379 (9817) (2012) 729-737.

[16] J. Lim, G. Cloete, D.T. Dunsmuir, B.A. Payne, C. Scheffer, P. von Dadelszen, et al., Usability and feasibility of PIERS on the Move: an mHealth app for pre-eclampsia triage, JMIR mHealth uHealth 3 (2) (2015) e37.

[17] B.A. Payne, J. Hutcheon, J.M. Ansermino, D. Hall, Z. Bhutta, S. Bhutta, et al., A risk prediction model for the assessment and triage of women with hypertensive disorders of pregnancy in low-resourced settings: the miniPIERS (Pre-eclampsia Integrated Estimate of RiSk) multi-country prospective cohort study, PLoS Med. 11 (1) (2014) e1001589.

[18] H.L. Nathan, A. de Greeff, N.L. Hezelgrave, L.C. Chappell, A.H. Shennan, An accurate semiautomated oscillometric blood pressure device for use in pregnancy (including pre-eclampsia) in a low-income and middle-income country population: the Microlife 3AS1-2, Blood Press Monit. 20 (1) (2015) 52-55.

[19] B.A. Payne, J.A. Hutcheon, D.T. Dunsmuir, G. Cloete, G. Dumont, D. Hall, et al., Assessing the incremental value of blood oxygen saturation ( $\mathrm{SpO} 2$ ) in the miniPIERS (Pre-eclampsia Integrated Estimate of RiSk) risk prediction model, J Obstet. Gynaecol. Can. 37 (1) (2015) 16-24.

[20] World Health Organization, 2012 WHO verbal autopsy instrument. https://www. who.int/healthinfo/statistics/verbalautopsystandards/en/index2.html, 2012.

[21] D. Rubin, Multiple Imputation for Nonresponse in Surveys, John Wiley and Sons, New York, 2004.

[22] World Health Organization, WHO recommendations on antenatal care for a positive pregnancy. https://apps.who.int/iris/bitstream/handle/10665/250796/ 9789241549912-eng. pdf;jsessionid = 3B5FE32AE497017E57AE2C7371169A8C? sequence $=1$, 2016 (accessed 2 April 2019).

[23] L. Magee, S. Sharma, H. Nathan, O. Adetoro, M. Bellad, S. Goudar, et al., The incidence of pregnancy hypertension in India, Pakistan, Mozambique, and Nigeria: A prospective population-level analysis, PLoS Med. 16 (4) (2019) e1002783.

[24] J.P. Souza, J.G. Cecatti, R.C. Pacagnella, T.M. Giavarotti, M.A. Parpinelli, R.S. Camargo, et al., Development and validation of a questionnaire to identify 
severe maternal morbidity in epidemiological surveys, Reprod. Health 7 (1) (2010) 16.

[25] The Alliance for Maternal and Newborn Health Improvement (AMANHI) study group. Population-based rates, timing, and causes of maternal deaths, stillbirths and neonatal deaths in south Asia and sub-Saharan Africa: a multi-country prospective cohort study. Lancet Glob Health 6(12) (2018) 1297-1308.

[26] S.B. Mazhar, A. Batool, A. Emanuel, A.T. Khan, S. Bhutta, Severe maternal outcomes and their predictors among Pakistani women in the WHO Multi-country Survey on Maternal and Newborn Health, Int. J. Gynaecol. Obstet. 129 (1) (2015) 30-33.

[27] National Institute of Population Studies, MEASURE Demographic \& Health Surveys,
ICF International. Pakistan Demographic and Health Survey 2012-13. https:// dhsprogram.com/pubs/pdf/FR290/FR290.pdf, 2013.

[28] Z. Sharafat, A survey of pregnancies complicated by antepartum haemorrhage, Med. Forum. Mon. 25 (2014) 9-13.

[29] S. Sheikh, S. Khokhar, P. Sirichand, R. Shaikh, A study of antepartum hemorrhage: maternal and perinatal outcomes, Med. Channel 16 (2) (2010) 268-271.

[30] Z. Bhutta, S. Soofi, S. Cousens, S. Mohammad, Z. Memon, I. Ali, et al., Improvement of perinatal and newborn care in rural Pakistan through community-based strategies: a cluster-randomised effectiveness trial, Lancet 377 (9763) (2011) 403-412. 